\title{
Aplicación del método de Nalgonda para la reducción de fluoruros de las fuentes de agua de las comunidades Atapo Culebrillas y Santa Teresa, Ecuador @(1) (8)
}

Application of the Nalgonda method for the reduction of fluorures of the water sources of the communities Atapo Culebrillas and Santa Teresa, Ecuador

Edwin Fernando Basantes Basantes. ${ }^{1}$, Anabel Cristina Cáceres del Salto. ${ }^{2}$, Cristian Javier Lozano Hernández. ${ }^{3} \&$ Laura Susana Cocha Telenchana. ${ }^{4}$

Recibido: 14-07-2019 / Revisado: 23-07-2019 /Aceptado: 15-08-2019/ Publicado: 10-09-2019

\begin{abstract}
DOI: https://doi.org/10.33262/cienciadigital.v3i3.4.872 Communities Atapo Culebrillas and Santa Teresa, in Guamote canton, Chimborazo province, have shown the presence of dental fluorosis, likely among other factors, because of drinking water conditions in these communities. Therefore, a characterization of the water from these sources identified fluoride ion as analyte, exceeding the permissible ceiling under Ecuadorian legislation TULSMA $(1.5 \mathrm{mg} / \mathrm{L})$, as suspect of the dental fluorosis presented in the area. A monitoring plan to quantify fluoride during 4 months was implemented, analyzes were performed at the Center for technical services and environmental technology transfer CESTTA, of the Escuela Superior Politécnica de Chimborazo. This research also proposed the Nalgonda method to reduce the fluoride content in the water, the most effective dose in reducing the fluoride content to safe limits for human health was found to be $3 \mathrm{~mL}$ of aluminium sulfate $0.075 \%$, plus $0.05 \mathrm{~g}$ of calcium carbonate, and 1 hour as settling time.
\end{abstract}

Keywords: Dental fluorosis, fluoride, characterization, analytes.

\section{Resumen}

En las comunidades Atapo Culebrillas y Santa Teresa del cantón Guamote de la provincia de Chimborazo se ha evidenciado la presencia de Fluorosis dental, es muy

\footnotetext{
1 Universidad Estatal Amazónica, Departamento Ciencias de la Tierra, Puyo, Ecuador, ef.basantesb@uea.edu.ec

2 Empresa pública metropolitana de gestión integral de residuos sólidos. EMGIRS-EP, Quito, Ecuador, anacris_09@hotmail.es

3 Universidad Técnica de Cotopaxi, Facultad de Ciencias Agropecuarias y Recursos Naturales, Carrera de Ingeniería Ambiental, Latacunga, Ecuador, cristian.lozano@utc.edu.ec

4 Escuela Superior Politécnica de Chimborazo, Facultad de Ciencias y Recursos Naturales, Carrera de Ingeniería Ambiental, Carrera de Agronomía, Francisco de Orellana, Ecuador, lcocha@espoch.edu.ec
} 
probable que la presencia de esta enfermedad entre otros factores se deba principalmente a las condiciones del agua que utilizan estas comunidades, por ello se realizó una caracterización del agua de estas fuentes, análisis que permitieron identificar al ión fluoruro como analito que sobrepasa el límite máximo permisible dictado por la legislación ecuatoriana TULSMA $(1.5 \mathrm{mg} / \mathrm{L})$ y como presunto responsable de la Fluorosis dental que presenta la zona. Se ejecutó un plan de monitoreo para la cuantificación de fluoruros durante 4 meses, los análisis se realizaron en el Centro de servicios técnicos y transferencia tecnológica ambiental CESTTA de la Escuela Superior Politécnica de Chimborazo, se propuso el método de Nalgonda para reducir el contenido de fluoruros en el agua, la dosis más efectiva para reducir el contenido de fluoruros a límites sin peligro para la salud humana que se encontró es de $3 \mathrm{~mL}$ de sulfato de aluminio al $0.075 \%$ mas $0,05 \mathrm{~g}$ de carbonato de calcio y como tiempo de sedimentación 1 hora.

Palabras clave: Fluorosis dental, fluoruros, Nalgonda, analitos.

\section{Introducción}

\section{El Fluor}

El Flúor es un elemento químico, su número atómico es el 9, situado en el grupo de los halógenos (grupo 17) de la tabla periódica de los elementos. Su símbolo es F, es un gas a temperatura ambiente, de color amarillo pálido, formado por moléculas diatómicas $F_{2}$. (2) El flúor se lo puede encontrar en las rocas, en combinación con minerales, rocas volcánicas, agua de mar, en los yacimientos de sal de origen marino, agua de los ríos, lagos, mares en concentraciones diversas. (3)

El flúor es el elemento esencial y componente importante en la estructura de huesos y dientes, está presente en forma natural en el agua de consumo humano. (2)

\section{Los fluoruros, el fluoruro de hidrógeno y el flúor}

Son sustancias químicamente relacionadas. El flúor es un gas natural de color amarillo pálido a verde de olor penetrante. Se combina con metales para producir fluoruros tales como el fluoruro de sodio y el fluoruro de calcio, ambos sólidos de color blanco. El fluoruro de sodio se disuelve fácilmente en agua, pero no así el fluoruro de calcio. El flúor también se combina con hidrógeno para producir fluoruro de hidrógeno, un gas incoloro. El fluoruro de hidrógeno se disuelve en agua formando ácido fluorhídrico (1).

\section{Ciclo del flúor}

El flúor no puede ser destruido en el ambiente; solamente puede cambiar de forma. El flúor forma sales con minerales en el suelo. El gas fluoruro de hidrógeno será absorbido por la lluvia, las nubes y la niebla y formará ácido fluorhídrico, el que caerá a la tierra. Los fluoruros liberados al aire por volcanes y la industria son transportados por el viento y la lluvia a aguas, suelo y fuentes de alimentos cercanas. En el agua y en el suelo los fluoruros se adhieren fuertemente al sedimento o a partículas en el suelo. 
La población general puede estar expuesta a los fluoruros en el aire, alimentos, agua potable y suelo contaminados (1).

\section{Toxicocinética del flúor}

\section{Absorción}

Las fuentes de flúor pueden ser inorgánicas y orgánicas. El aporte habitual de flúor a nuestro organismo es a partir de fluoruros inorgánicos. La solubilidad de estas sustancias no es uniforme, hay fluoruros solubles, como el fluoruro sódico $(\mathrm{NaF})$, el ácido fluorhídrico $(\mathrm{HF})$, que se absorben casi totalmente (75-90\%) y otros compuestos menos solubles como el fluoruro cálcico $\left(\mathrm{CaF}_{2}\right)$, el fluoruro magnésico $\left(\mathrm{MgF}_{2}\right)$ y el fluoruro de aluminio $\left(\mathrm{AlF}_{3}\right)$.

Dependiendo de su solubilidad, estos compuestos liberan iones de flúor, Los iones fluoruro de la dieta en forma de compuestos solubles, como el Fluoruro de sodio, se absorben rápidamente en una proporción del $80 \%$, Cantidades elevadas de iones de calcio, magnesio y aluminio reducen su absorción, al formar fluoruros menos solubles.

El flúor generalmente lo ingerimos como bebida, comida o como droga ( $\mathrm{NaF})$ en tabletas. Dependiendo de las características físico-químicas de sus componentes y de la solubilidad, una cantidad variable de la dosis de flúor ingerida será absorbida y alcanzará el torrente circulatorio.

El fluoruro se absorbe fundamentalmente en el estómago y en el intestino delgado, la absorción está relacionada con la acidez gástrica. Cuando el flúor en forma iónica $\left(\mathrm{F}^{-}\right)$entra en el estómago, se combina rápidamente con los iones hidrógeno $(\mathrm{H}+)$ y se convierte en ácido fluorhídrico $(\mathrm{HF})$, una molécula sin carga que atraviesa fácilmente las membranas biológicas, incluyendo la mucosa gástrica.

\section{Distribución}

El flúor se distribuye desde el plasma a todos los órganos y tejidos en función de la perfusión sanguínea que se produce en ellos. En general, la concentración de flúor presente en los tejidos blandos es baja. En el caso del riñón hay una concentración más alta que en el plasma, en cambio en el tejido adiposo y en el cerebro las concentraciones son menores. Existe una relación directa entre la concentración de flúor en la sangre materna y la fetal, las concentraciones de flúor en la sangre del cordón umbilical representan el $75 \%$ de la concentración en la sangre materna. El flúor que pasa al feto es rápidamente captado para la calcificación de huesos y dientes. (4)

Aproximadamente, el $99 \%$ de todo el flúor del organismo se encuentra en los tejidos calcificados (huesos y dientes). La cantidad acumulada en estos, depende de la cantidad ingerida, la duración de la exposición.

Los tejidos dentales experimentan una alta captación de flúor durante su desarrollo, pero la ausencia de remodelación y la mínima actividad metabólica después de la erupción, no permiten considerar un intercambio con el plasma, tal como se produce en el hueso. (4)

En la fase de maduración pre eruptiva el flúor presente en los fluidos que rodean la corona se incorpora a los tejidos mineralizados, dando lugar a fluorapatita y fluorhidroxiapatita. Este flúor incorporado es mayor en la zona externa del esmalte que en el interior y mayor en 
dientes permanentes que en temporales. El diente recién erupcionado sufre un proceso de des maduración posteruptiva que dura fundamentalmente dos años, durante el cual, si la concentración de iones flúor en la saliva y en la placa dental es elevada, se formarán cristales con mayor resistencia a la desmineralización.

\section{Excreción.}

El flúor se excreta por la orina, las heces, la saliva, el sudor y en menores cantidades por el pelo, la piel, y la leche (11).

El flúor eliminado por las heces corresponde a una pequeña fracción (10-15\%) del ingerido, que no ha sido absorbida en el intestino por su forma insoluble. La eliminación por el sudor es muy pequeña y está sometida a grandes variaciones climáticas e individuales, por lo que se considera prácticamente despreciable. En la saliva, se considera escaso su papel como vía de excreción del flúor, solo el 0.1-0,2\% del flúor ingerido aparece en saliva, la concentración de flúor en saliva varía dependiendo de la exposición previa a productos fluorados y del tiempo transcurrido. (5)

El flúor se elimina principalmente por orina. Se filtra por el glomérulo y se reabsorbe en los túbulos por difusión pasiva. La excreción renal se realiza de una forma relativamente rápida, una tercera parte del flúor absorbido aparece en orina a las 3-4 horas de absorbido, eliminándose casi totalmente en 12 horas. La excreción máxima se produce a las 1,5 - 3 horas de la ingestión.

La cantidad de flúor eliminada depende de diversos factores, entre los que se incluyen la edad del individuo, la composición de la dieta, la cantidad de orina excretada, el pH urinario, la integridad del riñón, la presencia de insuficiencia renal sobre todo si modifica la filtración glomerular, puede alterar el proceso de eliminación.

Durante el periodo de crecimiento la eliminación en orina es más baja, debido a que la cantidad de este ión que se está acumulando en los huesos y en los dientes aumenta.

El flúor que se excreta puede ser monitorizado además mediante otras pruebas biológicas. Los biomarcadores de flúor son valores que sirven para identificar el consumo deficiente o excesivo y la disponibilidad biológica del flúor. La OMS los define como: marcadores actuales (orina, plasma y saliva), marcadores recientes (uñas y pelo) y marcadores históricos (huesos y dientes). (5)

\section{Regulaciones de agua potable de la Agencia de protección Ambiental (EPA) para el fluoruro.}

En 1974, el Congreso aprobó la Ley de Agua Potable Segura. Esta ley requiere que la EPA determine el nivel de contaminantes en el agua potable a la que no tiene efectos adversos para la salud y no son probables de ocurrir.

Basadas únicamente en los posibles riesgos para la salud y la exposición durante toda la vida, con un margen de seguridad adecuado, se denomina nivel máximo de contaminante objetivo (MCLG). Los contaminantes son las sustancias químicas, físicas, biológicas o radiológicas o materia en el agua que pueden causar daño en la salud humana. (14) 
El MCLG para el fluoruro es de 4,0 $\mathrm{mg} / \mathrm{L}$ o $4.0 \mathrm{ppm}$. La EPA ha establecido este nivel de protección, basado en la mejor ciencia disponible para prevenir posibles problemas de salud. La EPA ha establecido una norma aplicable para el fluoruro, llamado un nivel máximo de contaminante (MCL), a 4,0 mg/L o $4.0 \mathrm{ppm}$. (14)

El nivel máximo de contaminante se establece tan cerca de los objetivos de salud como sea posible, teniendo en cuenta los costos, los beneficios y la capacidad de los sistemas públicos de agua para detectar y eliminar los contaminantes mediante tecnologías de tratamiento adecuados. (6).

La EPA también ha establecido una norma secundaria (SMCL) para el fluoruro en el 2,0 $\mathrm{mg} / \mathrm{L}$ o $2.0 \mathrm{ppm}$. Las normas secundarias son: Pautas no obligatorias sobre niveles de contaminantes que pueden causar efectos cosméticos (como la piel o decoloración de los dientes) o efectos estéticos (como el sabor, olor o color) en el agua potable. La EPA recomienda los estándares secundarios para los sistemas de agua. Sin embargo, los estados pueden optar por adoptar como normas de obligado cumplimiento.

La decoloración de los dientes es causada por exposiciones excesivas de fluoruro durante el período de formación antes de la erupción de los dientes en los niños. El estándar secundario de 2,0 $\mathrm{mg} / \mathrm{L}$ pretende ser una guía para un nivel de límite superior en las áreas que tienen altos niveles de fluoruro que ocurre naturalmente. El nivel de la SMCL se estableció sobre la base de un balance de los efectos beneficiosos de protección contra las caries y los efectos indeseables de las exposiciones excesivas que conduzcan a la decoloración. Los Estados pueden establecer normas más estrictas para el fluoruro que los tomados por la EPA (14).

\section{Métodos disponibles para la eliminación del fluoruro del agua potable}

- Métodos de precipitación-floculación.

- Métodos de adsorción.

- Procesos que implican la evaporación del agua.

- Procesos basados en tecnologías de membranas.

Los métodos de los tres primeros grupos engloban distintas técnicas según se trate de aplicaciones industriales o de sistemas domésticos. Por ejemplo, los procesos que implican evaporación del agua van desde las técnicas de desalación basadas en la utilización de energía térmica o solar hasta los destiladores solares unifamiliares, que son pequeños recipientes cubiertos de plástico o vidrio que recoge y canaliza el agua evaporada en su interior hacia un depósito de consumo (12).

Los métodos basados en la tecnología de membranas no suelen considerarse adecuados para países en desarrollo, pero pueden representar una alternativa viable para instalaciones de tamaño medio.

Los métodos más utilizados en los países en desarrollo son los de precipitación-floculación, que pueden adoptar formas caseras, como la precipitación sobre carbón vegetal o animal o formas semi industriales como el método Nalgonda y sus variantes basadas en la precipitación química con reactivos cálcicos, que es el método convencional para la eliminación de fluoruros en aguas. 
Si la precipitación se complementa con una floculación-sedimentación utilizando sales de aluminio (sulfato de aluminio hidratado), se obtiene una concentración de fluoruro suficientemente baja como para que el agua pueda ser sometida a un proceso de potabilización (1-2 $\mathrm{mg} / \mathrm{L})$.

La técnica se denomina Nalgonda y fue desarrollada originalmente por el National Environmental Engineering Research Institute de India (NEERI) para su uso en pequeñas instalaciones comunitarias o familiares. El proceso tiene lugar mediante la adición, a la vez o sucesivamente, de carbonato de calcio y sulfato de aluminio en un tanque. En las condiciones alcalinas obtenidas tras la adición del carbonato, el sulfato de aluminio forma flóculos de hidróxido de aluminio $\mathrm{Al}(\mathrm{OH})_{3}$. Durante la sedimentación, los flóculos arrastran el fluoruro y otros iones mediante fuerzas electrostáticas. El tiempo necesario para la sedimentación de los flóculos es de unas cuatro horas. El pH debe de estar en el rango 5-8 para evitar que el aluminio pase a la disolución en diversas formas $\left(\mathrm{Al}(\mathrm{OH})_{2}+, \mathrm{Al}(\mathrm{OH})_{4^{-}}\right)$, la ventaja principal se basa en su bajo coste (12).

Factores que complican el método de precipitación-floculación, es la excesiva salinidad del agua, que aumentaría con el tratamiento, por lo que no es recomendable para aguas con sólidos totales disueltos por encima de $1500 \mathrm{mg} / \mathrm{L}$. Tampoco da buenos resultados para aguas con más de $20 \mathrm{mg} / \mathrm{L}$ de fluoruro.

\section{Metodología}

\section{Determinación de Fluoruros}

La determinación de Fluoruros $\left(\mathrm{F}^{-}\right)$se la realizó mediante el método espectrofotométrico STANDAR METHODS 4500 D (2012), se utilizó Espectrofotómetro (HACH DR/2800), se realizó la verificación del equipo mediante la lectura de un estándar de $\mathrm{F}^{-}$de $2 \mathrm{mg} / \mathrm{L}$, se tomó $10 \mathrm{~mL}$ de agua desionizada para el blanco y $10 \mathrm{~mL}$ de muestra, se colocaron en celdas diferentes, se añadió $2 \mathrm{~mL}$ reactivo de Spands al blanco y a la muestra, se agitó por 10 seg y se esperó $1 \mathrm{~min}$ como periodo de reacción, se enceró el equipo con la celda del blanco y se leyó la muestra a una longitud de onda de $580 \mathrm{~nm}$.

\section{Determinación de Turbidez.}

La determinación de Turbidez se la realizó mediante el método nefelométrico EPA 180.1, se utilizó turbidímetro $(\mathrm{HACH})$, se realizó la verificación del equipo mediante la lectura de un estándar de turbiedad de 16 NTU, se tomó $10 \mathrm{~mL}$ de agua desionizada para el blanco, se colocó en el receptáculo del equipo y se procedió a encerarlo, se añadió $10 \mathrm{~mL}$ de muestra a una celda y se realizó la lectura.

\section{Cuantificación del Analito que excede los límites establecidos por la normativa}

Se cuantifico el ión fluoruro durante los meses de junio a septiembre del 2013, se encontró que existe diferencia significativa entre las dos comunidades en estudio en la concentración de fluoruros. 
Tabla 1. Cuantificación de $\mathrm{F}^{-}$del agua de las fuentes de abastecimiento de las comunidades Atapo Culebrillas y Santa Teresa, durante los meses de junio a septiembre del 2013.

\begin{tabular}{|l|r|c|l|l|}
\hline Sitio de estudio & \multicolumn{2}{|c|}{ N de muestras } & F- mg/L & $\delta$ \\
\hline Atapo Culebrillas & 31 & $\mathrm{n}=2$ & 2.66 & 0.17 \\
\hline Santa Teresita & 31 & $\mathrm{n}=2$ & 2.32 & 0.22 \\
\hline
\end{tabular}

$\mathrm{n}=$ Número de repeticiones

Fuente: Elaboración propia

\section{Alternativa para controlar la contaminación de las fuentes.}

Pruebas del Método de Nalgonda con variación carbonato de calcio y la concentración de sulfato de aluminio.

Con ayuda de una prueba de jarras se aplicó el método de Nalgonda para reducir el nivel de fluoruros en el agua de las comunidades de estudio, se observa las distintas variaciones en la concentración de sulfato de aluminio $\left(\mathrm{Al}_{2}\left(\mathrm{SO}_{4}\right)_{3}\right)$ y la adición de carbonato de calcio $\left(\mathrm{CaCO}_{3}\right)$, como también los resultados obtenidos de la aplicación de las distintas dosis de reactivos. (Tabla 2 y 3 ).

Tabla 2. Variación de la concentración de $5 \mathrm{~mL}$ de $\mathrm{Al}_{2}\left(\mathrm{SO}_{4}\right)_{3}$ con respecto a una adición de $0,05 \mathrm{~g} \mathrm{CaCO}_{3}$ a $2 \mathrm{~L}$ de agua problema en $1 \mathrm{~h}$ como tiempo de sedimentación.

\begin{tabular}{|c|c|c|c|c|c|c|}
\hline \multicolumn{7}{|c|}{ Resultados de la aplicación de la dosis } \\
\hline \multirow{2}{*}{$\begin{array}{c}\mathrm{V} . \\
\mathrm{Al}_{2}\left(\mathrm{SO}_{4}\right)_{3}\end{array}$} & \multirow[b]{2}{*}[]{. $\mathrm{Al}_{2}\left(\mathrm{SO}_{4}\right)_{3}$} & \multirow[b]{2}{*}{$\mathrm{CaCO}_{3}$} & \multicolumn{2}{|c|}{ Atapo Culebrillas } & \multicolumn{2}{|c|}{ Santa Teresa } \\
\hline & & & $\mathrm{pH}$ & F- & $\mathrm{pH}$ & F- \\
\hline $\mathrm{mL}$ & $\%$ & $\mathrm{~g}$ & uni $\mathrm{pH}$ & $\mathrm{mg} / \mathrm{L}$ & uni $\mathrm{pH}$ & $\mathrm{mg} / \mathrm{L}$ \\
\hline \multirow{4}{*}{5} & 0,025 & \multirow{4}{*}{0.05} & 7,8 & 2,52 & 7,83 & 2,16 \\
\hline & 0,05 & & 7,72 & 1,83 & 7,71 & 1,79 \\
\hline & 0,075 & & 7,53 & 0,81 & 7,62 & 0,80 \\
\hline & 0,1 & & 7,48 & 0,69 & 7,54 & 0,66 \\
\hline
\end{tabular}

Fuente: Elaboración propia

Tabla 3. Variación de la concentración de $5 \mathrm{~mL}$ de $\mathrm{Al}_{2}\left(\mathrm{SO}_{4}\right)_{3}$ con respecto a una adición de $0,10 \mathrm{~g} \mathrm{CaCO}_{3}$ a $2 \mathrm{~L}$ de agua problema en $1 \mathrm{~h}$ como tiempo de sedimentación.

\begin{tabular}{|c|c|c|c|c|c|c|}
\hline \multicolumn{7}{|c|}{ Resultados de la aplicación de la dosis } \\
\hline \multirow[b]{2}{*}{ V. $\mathrm{Al}_{2}\left(\mathrm{SO}_{4}\right)_{3}$} & \multirow[b]{2}{*}{ []. $\mathrm{Al}_{2}\left(\mathrm{SO}_{4}\right)_{3}$} & \multirow[b]{2}{*}{$\mathrm{CaCO}_{3}$} & \multicolumn{2}{|c|}{ Atapo Culebrillas } & \multicolumn{2}{|c|}{ Santa Teresa } \\
\hline & & & $\mathrm{pH}$ & F- & $\mathrm{pH}$ & F- \\
\hline $\mathrm{mL}$ & $\%$ & $\mathrm{~g}$ & uni pH & $\mathrm{mg} / \mathrm{L}$ & uni $\mathrm{pH}$ & $\mathrm{mg} / \mathrm{L}$ \\
\hline \multirow{4}{*}{$5 \mathrm{~mL}$} & 0,025 & \multirow{4}{*}{0.10} & 7,99 & 2,44 & 7,71 & 2,08 \\
\hline & 0,05 & & 7,85 & 1,78 & 7,68 & 1,75 \\
\hline & 0,075 & & 7,73 & 0,80 & 7,56 & 0,79 \\
\hline & 0,1 & & 7,64 & 0,66 & 7,39 & 0,64 \\
\hline
\end{tabular}

Fuente: Elaboración propia 


\section{Pruebas del Método de Nalgonda con variación del volumen de sulfato de aluminio y el tiempo}

Se muestra la variación del volumen de sulfato de aluminio y los resultados obtenidos en la concentración de fluoruros (Tabla 4).

Tabla 4. Variación del volumen de $\mathrm{Al}_{2}\left(\mathrm{SO}_{4}\right)_{3}$ con respecto a una adición de $0,05 \mathrm{~g} \mathrm{CaCO}_{3} \mathrm{a}$ $2 \mathrm{~L}$ de agua problema en $1 \mathrm{~h}$ como tiempo de sedimentación.

\begin{tabular}{ccccccc}
\hline & \multicolumn{5}{c}{ Resultados de la aplicación de la dosis } \\
V. $\mathrm{Al}_{2}\left(\mathrm{SO}_{4}\right)_{3}$ & {$\left[\mathrm{C} \cdot \mathrm{Al}_{2}\left(\mathrm{SO}_{4}\right)_{3}\right.$} & $\mathrm{CaCO}_{3}$ & $\mathrm{AH}$ & $\mathrm{F}-$ & $\mathrm{pH}$ & $\mathrm{F}-\mathrm{mg} / \mathrm{L}$ \\
$\mathrm{mL}$ & $\%$ & $\mathrm{~g}$ & $\mathrm{uni} \mathrm{pH}$ & $\mathrm{mg} / \mathrm{L}$ & uni $\mathrm{pH}$ & $\mathrm{mg} / \mathrm{L}$ \\
\hline 2 & & & 7,57 & 1.88 & 7,42 & 1,97 \\
$\mathbf{3}$ & & & 7,49 & 0.84 & 7,4 & 0.82 \\
4 & 0,075 & 0,05 & 7,42 & 0.71 & 7,35 & 0,66 \\
5 & & & 7,33 & 0.68 & 7,28 & 0,65 \\
\hline
\end{tabular}

Nota: $\mathrm{V}=$ volumen $\quad[]=$ Concentración $\quad \%=$ porcentaje $\quad \mathrm{g}=$ gramo

Fuente: Elaboración propia

En la (Tabla 5) se muestra los resultados obtenidos al variar el tiempo de sedimentación luego de aplicar el método de Nalgonda.

Tabla 5. Variación del tiempo con respecto a la concentración de F-

\begin{tabular}{|c|c|c|c|c|c|c|c|}
\hline \multicolumn{8}{|c|}{ Resultados de la aplicación de la dosis } \\
\hline \multirow{2}{*}{$\begin{array}{l}\mathrm{V} \\
\mathrm{Al}_{2}\left(\mathrm{SO}_{4}\right)_{3}\end{array}$} & \multirow{2}{*}[]{$\cdot \mathrm{Al}_{2}\left(\mathrm{SO}_{4}\right)_{3}$} & \multirow{2}{*}{$\mathrm{CaCO}_{3}$} & \multicolumn{3}{|c|}{$\begin{array}{c}\text { Atapo } \\
\text { Culebrillas }\end{array}$} & \multicolumn{2}{|c|}{ Santa Teresa } \\
\hline & & & $\mathrm{t}$ & $\mathrm{pH}$ & $\begin{array}{c}\mathrm{F}- \\
\mathrm{mg} / \mathrm{L}\end{array}$ & $\mathrm{pH}$ & $\begin{array}{c}\mathrm{F}- \\
\mathrm{mg} / \mathrm{L}\end{array}$ \\
\hline $\mathrm{mL}$ & $\%$ & G & $\mathrm{h}$ & $\begin{array}{l}\text { uni } \\
\mathrm{pH}\end{array}$ & $\mathrm{mg} / \mathrm{L}$ & $\begin{array}{l}\text { uni } \\
\mathrm{pH}\end{array}$ & $\mathrm{mg} / \mathrm{L}$ \\
\hline \multirow{4}{*}{3} & \multirow{4}{*}{0,075} & \multirow{4}{*}{0,05} & 1 & 7.57 & 0.84 & 7.49 & 0.82 \\
\hline & & & 2 & 7.47 & 0.71 & 7.40 & 0.68 \\
\hline & & & 3 & 7.47 & 0.69 & 7.40 & 0.68 \\
\hline & & & 4 & 7.47 & 0.69 & 7.40 & 0.66 \\
\hline
\end{tabular}

Nota: $\mathrm{V}=$ volumen $\quad[]=$ Concentración $\%=$ porcentaje $\quad \mathrm{g}=$ gramo

Fuente: Elaboración propia 


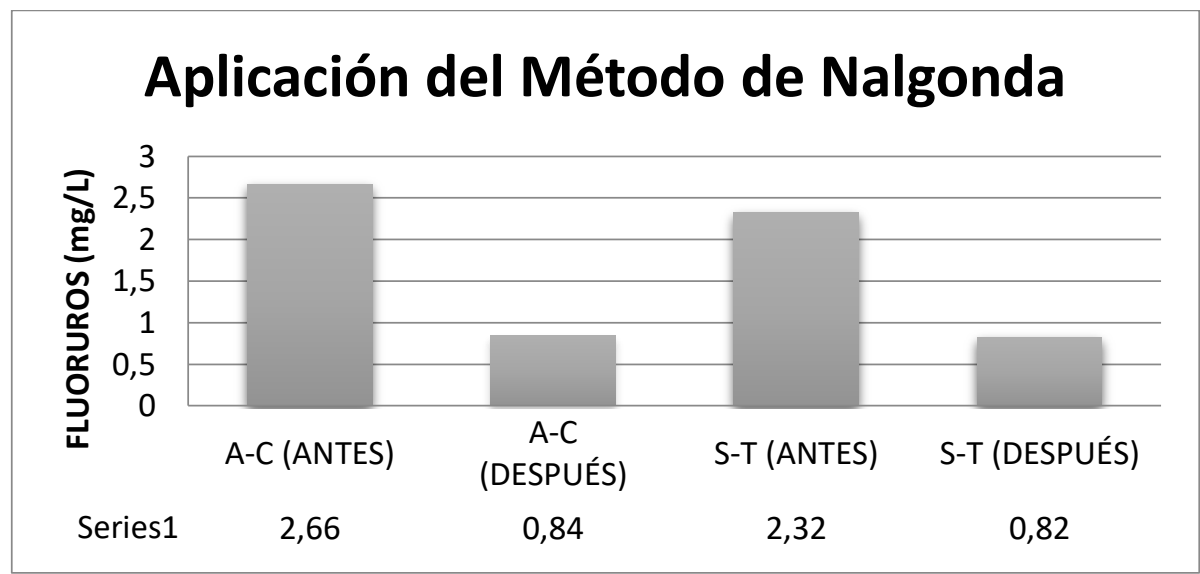

Figura 1. Aplicación del método de Nalgonda al agua de las fuentes de abastecimiento de la comunidad Atapo Culebrillas y Santa Teresa.

Nota: $\mathrm{A}-\mathrm{C}=$ Comunidad Atapo Culebrillas $\mathrm{S}-\mathrm{T}=$ Comunidad Santa Teresa

Fuente: Elaboración propia

\section{Discusión}

\section{Concentración de fluoruros durante los 4 meses de monitoreo}

Los niveles reportados de fluoruros durante los 4 meses de monitoreo dieron una media de 2,66 mg/L en la comunidad de Atapo Culebrillas, mientras que en la comunidad de Santa Teresa dieron una media de 2,32mg/L valores que sobrepasaron los niveles normales dictados por el TULSMA (LMP F $\left.{ }^{-}=1,5 \mathrm{mg} / \mathrm{L}\right)$. (13).

A su vez estos valores sobrepasaron la normativa de la EPA norma secundaria (SMCL) que reporta un límite máximo de $2 \mathrm{mg} / \mathrm{L}$ y de la Organización Mundial de la Salud (OMS) que reporta un límite máximo de 1,5 mg/L.

La prueba z-normal que se realizó a un nivel de confianza del $95 \%$ demostró que existe una diferencia significativa entre las medias de las concentraciones de fluoruros entre la comunidad Atapo Culebrillas y la comunidad Santa Teresa ya que se obtuvo un z tabulado= 1,96 y un z experimental $=5,57$ con esto se rechaza la $\mathrm{H}_{0}=$ Las medias de las concentraciones $\left(\mathrm{F}^{-} \mathrm{mg} / \mathrm{L}\right)$ de las comunidades Atapo Culebrillas y de Santa Teresa NO son estadísticamente diferentes y se acepta $\mathrm{H}_{1}=\mathrm{Las}$ medias de las concentraciones $\left(\mathrm{F}^{-} \mathrm{mg} / \mathrm{L}\right)$ de las comunidades Atapo Culebrillas y de Santa Teresa son significativamente diferentes, por esta razón se realiza la prueba de jarras individualmente para cada comunidad.

Estudios realizados por la USEPA en su artículo Acharya, S. 2005. Dental caries, its surface susceptibility and dental fluorosis in South India. International Dental Journal, determinaron que, a una concentración del agua de consumo de 1,22ppm $\mathrm{F}^{-}$existe una prevalencia del 54,3 $\%$ de Fluorosis dental y a una concentración del 3,41ppm $\mathrm{F}^{-}$existe una prevalencia de Fluorosis dental del $100 \%$. (8) 
Comparando los niveles de Fluoruros de este estudio con los encontrados en las dos comunidades Atapo Culebrillas y Santa Teresa, se puede decir que el Fluoruro es el posible responsable de la Fluorosis dental que presenta la zona de estudio.

Estudios realizados por Kotecha et al. (2012) en el distrito Gujarat India muestran que a una mayor concentración de fluoruros en el agua de consumo (1.92-.4.10) $\mathrm{mg} / \mathrm{L}$ aumenta la prevalencia de Fluorosis dental en la población (59.31\%) y recomienda reducir el contenido de fluoruro del agua potable o buscar fuentes alternativas para el suministro de agua con menor contenido de fluoruro. (9)

Montero et al. (2007) en estas mismas líneas de estudio encontró que con una concentración de $1.03+0.23 \mathrm{ppm}$ de fluoruros en el agua de consumo existe una prevalencia del $81.9 \%$ de fluorosis dental. (10)

\section{Método de Nalgonda para descender los niveles de Fluoruros en el agua de las fuentes.}

En la (Tabla 2) se observa una adición de $5 \mathrm{~mL}$ de sulfato de aluminio a cuatro diferentes concentraciones $(0,025,0,050,0,075,1,00) \%$ contra una adición de $0,05 \mathrm{~g}$ a $2 \mathrm{~L}$ de agua problema y una hora como tiempo de sedimentación, y en la (Tabla 3) se varia la concentración de carbonato de calcio a $0,10 \mathrm{~g}$, comparando las dos tablas se escogió como dosis adecuada para continuar con la prueba la adición de $5 \mathrm{~mL}$ se sulfato de aluminio al 0,075\% y $0.05 \mathrm{~g}$ de carbonato de calcio ya que a esta concentración se tiene un nivel de $\mathrm{F}^{-}$de $0,81 \mathrm{mg} / \mathrm{L}$ en la comunidad Atapo Culebrillas y $0,80 \mathrm{mg} / \mathrm{L}$ en la comunidad Santa Teresa encontrándose dentro de los niveles establecidos por legislación nacional e internacional.

No se escoge la adición de $5 \mathrm{~mL}$ se sulfato de aluminio al 0,075 \% y una adición de $0.10 \mathrm{~g}$ de carbonato de calcio dando una concentración de $\mathrm{F}^{-}$de $0,80 \mathrm{mg} / \mathrm{L}$ y $0.79 \mathrm{mg} / \mathrm{L}$ en las comunidades Atapo Culebrillas y Santa Tersa respectivamente, debido a que se ocupa el doble en peso de carbonato de calcio para llegar a un contenido similar de fluoruros, y a su vez esto encarecería los costos del tratamiento.

En la (Tabla 4) se observa una adición de cuatro volúmenes diferentes de sulfato de aluminio $(2,3,4,5) \mathrm{mL}$ al $0,075 \%$ contra una adición de $0,05 \mathrm{~g}$ a $2 \mathrm{~L}$ de agua problema y una hora como tiempo de sedimentación, comparando los datos en la tabla se escogió como dosis adecuada $3 \mathrm{~mL}$ se sulfato de aluminio al $0,075 \%$ y $0.05 \mathrm{~g}$ de carbonato de calcio para continuar con la prueba, ya que a esta concentración se tiene un nivel de $\mathrm{F}^{-}$en Atapo Culebrillas $(0,84 \mathrm{mg} / \mathrm{L})$ y Santa Teresa $(0,82 \mathrm{mg} / \mathrm{L})$ encontrándose dentro de los niveles establecidos por legislación.

No se escoge la adición de 4 o $5 \mathrm{~mL}$ se sulfato de aluminio debido a que el nivel de $\mathrm{F}^{\text {es }}$ similar al de la adición de $3 \mathrm{~mL}$, y a su vez la adición de un volumen mayor encarecería los costos del tratamiento. 
En la (Tabla 5) se observa una adición de $3 \mathrm{~mL}$ de sulfato de aluminio al 0,075\% contra una adición de $0,05 \mathrm{~g}$ a $2 \mathrm{~L}$ de agua problema y un tiempo de sedimentación de $(2,3,4)$ horas comparando los datos en la tabla se escogió como dosis adecuada estipulada en la (Tabla 4) que ocupa $3 \mathrm{~mL}$ se sulfato de aluminio al $0,075 \%$ y $0.05 \mathrm{~g}$ de carbonato de calcio en una hora como tiempo de sedimentación.

No se escoge el tiempo de espera de $(2,3,04)$ horas debido a que se perdería mucho tiempo en el tratamiento de la muestra para obtener un resultado similar.

\section{Conclusiones.}

- Al cuantificar los diferentes analitos en las muestras en cuestión, se encontró que la concentración de fluoruros excede los límites máximos permisibles establecidos por las normativas tanto nacionales TULSMA $(1.5 \mathrm{mg} / \mathrm{L})$ e internacionales USEPA $(2 \mathrm{mg} / \mathrm{L})$ y OMS $(1,5 \mathrm{mg} / \mathrm{L})$

- La cuantificación del ión fluoruro durante el periodo de muestreo mostró diferencia significativa entre las dos comunidades.

- Mediante el método de Nalgonda y con la ayuda de una prueba de jarras se determinó que con una adición de $3 \mathrm{~mL}$ de sulfato de aluminio al 0,075\% y $0,05 \mathrm{~g}$ de carbonato de calcio en un tiempo de 1 hora para sedimentación aplicada a 2L de muestra es la concentración más adecuada para reducir los niveles de fluoruros en Las fuentes de abastecimiento de agua de las comunidades Atapo culebrillas y Santa Teresa a niveles normales $(<1.5 \mathrm{mg} / \mathrm{L})$.

\section{Referencias bibliográficas.}

1. ATSDR, (2003). Agencia para Sustancias Tóxicas y el Registro de Enfermedades División de Toxicología y Medicina Ambiental. Fluorides, Hydrogen Fluoride and Fluorine.

2. Beltrán, M (2012). Investigar las consecuencias del efecto acumulativo del flúor, una necesidad imperante de la profesión odontológica. Revista Colombiana de Investigación en Odontología. Vol. 3 N 7.

3. Capo, M (2007). Principios de Ecotoxicología. Madrid, Tebar.

4. EPA. (2008). Fluoride: Dose-Response Analysis for Non-cancer Effects, FluorideRelated Skeletal Effects: Evaluations of Key Studies, Health and Ecological Criteria Division Office of Water.

5. Firempong et al., (2013). Soluble fluoride levels in drinking water-a major risk factor of dental fluorosis among children in Bongo community of Ghana. Ghana Medical Journal. Vol. 47 Issue 1, p16-23. 8p. 
6. Gómez, R (2010). Fluorosis dental en estudiantes de 8 a 12 años de la escuela fiscal mixta "Luis Vivero Espinoza" de la parroquia Totoras en la ciudad de Ambato. QuitoEcuador. Tesis de grado UCE.

7. Husain et al., (2013) Assessment of Fluoride Level in Groundwater and Prevalence of Dental Fluorosis in Didwana Block of Nagaur District, Central Rajasthan, India. International Journal of Occupational \& Environmental Medicine. Vol. 4 Issue 4, p178-184. 7p. 1 Chart, 2 Graphs.

8. IRIS USEPA (2012). U.S. Environmental Protection. Agency Fluoride, Integrated Risck Imformation System.

9. Kotecha et al., (2012). Prevalece of dental fluorosis \& dental caries in association with high levels of drinking water fluoride content in a district of Gujarat, India. Indian Journal of Medical Research. Vol. 135 Issue 6, p873- 877. 5p.

10. Montero et al., (2007). Experiencia de caries y fluorosis dental en escolares que consumen agua con diferentes concentraciones de fluoruro en Maiquetía Estado Vargas Venezuela.

11. REPETTO, M. (2009). Toxicología Fundamental (4ta ed.). España: Edición Días de Santos.

12. Roberto, R. (2007). Eliminación de Fluoruros del agua potable, Grupo de Ingeniería Química, Universidad de Alcalá.

13. Texto Unificado de Legislación Ambiental Secundaria TULSMA libro VI anexo I, tabla 1.

14. USEPA (2013). U.S. Environmental Protección Agency Basic Information about Fluoride in Drinking

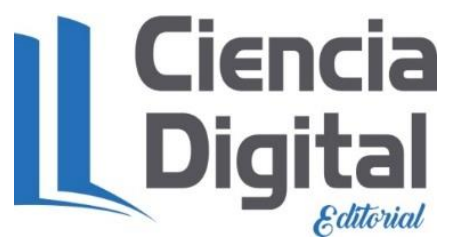


PARA CITAR EL ARTÍCULO INDEXADO.

Basantes Basantes, E., Cáceres del Salto, A., Lozano Hernández, C., \& Cocha Telenchana, L. (2019). Aplicación del método de Nalgonda para la reducción de fluoruros de las fuentes de agua de las comunidades Atapo Culebrillas y Santa Teresa, Ecuador. Ciencia Digital, 3(3.4.), 276-288. https://doi.org/10.33262/cienciadigital.v3i3.4.872

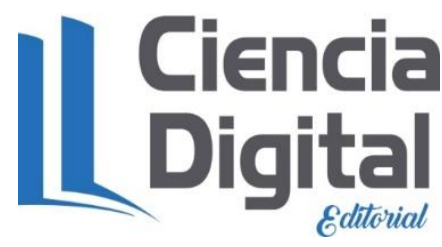

El artículo que se publica es de exclusiva responsabilidad de los autores y no necesariamente reflejan el pensamiento de la Revista Ciencia Digital.

El artículo queda en propiedad de la revista y, por tanto, su publicación parcial y/o total en otro medio tiene que ser autorizado por el director de la Revista Ciencia Digital.
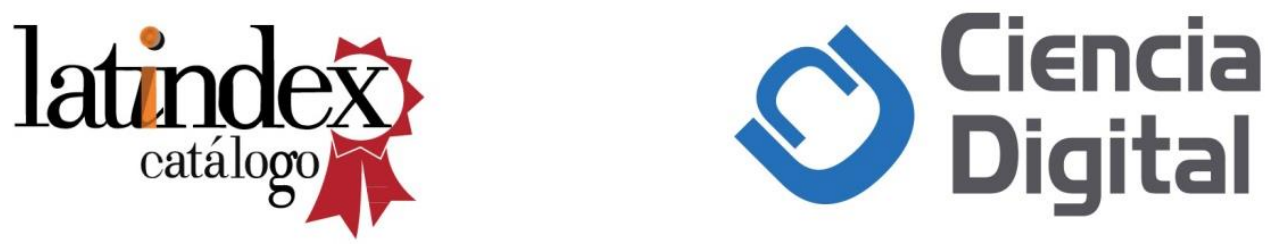